\title{
Contrôle génétique de l'hydroxylation en 5' des anthocyanes dans les fleurs d'Hedysarum coronarium L et $\boldsymbol{H}$ capitatum Desf, Asch et $\mathrm{Gr}$
}

\author{
A Chriki \\ École normale supérieure de bizerte, 7021 Zarzouna, Tunisie
}

(Reçu le 6 février 1990; accepté le 11 juin 1990)

\begin{abstract}
Résumé - L'analyse génétique et chromatographique de la pigmentation florale chez $\mathrm{H}$ coronarium et $\mathrm{H}$ capitatum montre que l'introduction du groupement hydroxyle en position $5^{\prime}$ de la molécule anthocyanique est déterminée par deux gènes indépendants $V$ et $R$. Les allèles dominants de ces gènes contrôlent la synthèse de la malvidine. Cependant, l'action du gène $R$ est moins prononcée, de sorte que :

- dans les lignées VVRR (ou VVrr), l'hydroxylation quasi totale en 5 ' des anthocyanidines détermine l'accumulation de la malvidine et l'expression du phénotype violet;

- dans les lignées vvRR, l'hydroxylation partielle en 5 ' des anthocyanidines entraîne l'accumulation simultanée de la malvidine et de la paeonidine, déterminant le phénotype rouge-violacé;

- dans les lignées homozygotes récessives (vvrr), l'hydroxylation en 5' des anthocyanidines n'a pas lieu et la paeonidine est synthétisée en abondance dans les fleurs rouges.
\end{abstract}

contrôle génétique / anthocyane / hydroxylation en $5^{\prime}$ / Hedysarum

Summary - The genetic control of anthocyanin $5^{\prime}$ hydroxylation in flowers of Hedysarum coronarium $L$ and $\boldsymbol{H}$ capitatum Desf, Asch et Gr. The results of chemogenetic studies on flower pigmentation in Hedysarum coronarium and $\mathrm{H}$ capitatum are reported. Using thin-layer chromatography for the identification of anthocyanidins in parental strains with different flower colorations, 3 phenotypes were clearly defined (table II) :

- The red phenotype is determined by the accumulation of paeonidin, a pigment derived from cyanidin (3'hydroxylated: fig 1).

- The purple flowers accumulate malvidin derived from delphinidin $\left(3^{\prime}, 5^{\prime}\right.$-dihydroxylated : fig 1).

- The purplish-red phenotype results from the simultaneous accumulation of malvidin and paeonidin.

The chromatographic study of pigments in different colored strains was confirmed by semi-quantitative determination of anthocyanidins in defined phenotypes (table III). We concluded that the flower color type in Hedysarum was correlated with flavonoid 5' hydroxylation activity.

Segregation analysis of flower colors in $F_{2}$ (table VI), showed that the introduction of the 5'-hydroxyl group of the anthocyanidin molecule was determined by 2 particular genes, $V$ and $R$ (table VII). Dominant alleles of these genes control the synthesis of malvidin. However, the action of the gene $R$ is less pronounced, so that :

- In lines VVRR (or VVrr), the quasi-total anthocyanidin 5'-hydroxylation determines the accumulation of malvidin and the expression of purple phenotype.

- In lines wRR, the partial anthocyanidin 5'-hydroxylation is correlated with the simultaneous accumulation of malvidin and paeonidin, determining the purplish-red phenotype.

- In homozygous recessive lines (wrr), anthocyanidin 5'-hydroxylation does not occur and paeonidin is abundantly synthesized in red flowers.

genetic control / anthocyanin / 5'-hydroxylation / Hedysarum 


\section{INTRODUCTION}

Depuis quelques années, des espèces du genre Hedysarum (légumineuses, papilionacées) poussant dans le bassin méditérranéen et en particulier en Afrique du Nord, font l'objet de différentes recherches. Ces travaux ont porté essentiellement sur l'étude de la biologie de la reproduction (Chriki et al, 1984) ainsi que sur la variabilité tant morphologique (Combes et al, 1975; Figier et al, 1978) qu'enzymatique (Trifi-Farah et al, 1989a, b). Le polymorphisme de l'ADN chloroplastique et mitochondrial a été également analysé dans le genre Hedysarum (Baatout et al, 1985). Une analyse cytogénétique préliminaire (Combes et al, 1975; Baatout et al, 1985) montre qu'à l'exception d' $H$ pallidum (tétraploïde), les espèces d'Hedysarum étudiées sont diploïdes $(2 n=16)$.

Par ailleurs, des études de variabilité, d'H coronarium notamment, ont porté sur des caractères agronomiques, afin de créer de nouveaux cultivars (Monotti, 1975; Sarno et al, 1978). La valeur nutritive $\mathrm{d}^{\prime} H$ coronarium a été étudiée, entre autres, par Cenni et al (1968) et Ballatore (1972). Ces travaux ont montré que l'espèce $H$ coronarium présentait des qualités fourragères équivalentes à celles du trèfle violet et de la luzerne et ont permis la création de variétés cultivées.

Cependant, la digestibilité et la valeur nutritive des écotypes spontanés d'Hedysarum paraissent meilleures que celles des variétés cultivées (Cenni et al, 1968). C'est pourquoi, la plupart des travaux effectués et ceux en cours de réalisation en Tunisie sont axés sur les types sauvages afin d'inventorier les ressources génétiques des espèces du genre Hedysarum. Dans ce cadre, une étude détaillée des populations naturelles en vue de leur évaluation s'est avérée nécessaire tant du point de vue fondamental que du point de vue agronomique. Pour réaliser cet objectif, il était important d'identifier des marqueurs génétiques, tels que ceux liés à la coloration anthocyanique des fleurs.

De nombreuses prospections effectuées en Tunisie $(1975,1981,1983)$, au Sud de la France (1976), au Maroc (1977), en Algérie (1981), en Sardaigne et à Malte (1983) ont montré que les populations naturelles d' $\mathrm{H}$ coronarium et d' $\mathrm{H} \mathrm{Ca}$ pitatum offraient une diversité de coloration des fleurs allant du rouge au violet, en passant par le rouge-violacé.

C'est à partir de graines récoltées dans les populations naturelles que nous avons constitué, par des autofécondations répétées, diverses lignées, à fleurs rouges ou violettes chez $\mathrm{H}$ coronarium, à fleurs rouge-violacées ou violettes chez $H$ capitatum. L'obtention de telles lignées stables quant à leur pigmentation florale a nécessité, au moins, trois générations successives d'autofécondation. Ces lignées ont été utilisées dans l'étude de l'hérédité de la pigmentation anthocyanique chez ces deux taxons.

\section{MATÉRIEL ET MÉTHODES}

\section{Les lignées}

Les principales caractéristiques des lignées utilisées dans cette étude sont indiquées dans le tableau I. Les autofécondations et les croisements ont été réalisés selon les techniques habituelles, adaptées à la biologie florale d'Hedysarum (Chriki et al, 1982 et 1984).

\section{Appréciation quantitative des anthocyanidines}

Comme la plupart des flavonoïdes, les anthocyanes se trouvent dans la vacuole sous forme hétérosidique. Leur hydrolyse acide libère le (ou les) sucre(s) et l'aglycone ou anthocyanidine (Harbone, 1973). Les principaux flavonoïdes non colorés (flavones et flavonols) et les anthocyanes ont été déjà identifiés chez Hedysarum (Chriki et al, 1982; Chriki et Harborne, 1983; Chriki et al, 1986).

Tableau I. Origine et phénotype des lignées utilisées dans l'étude de la pigmentation florale chez $H$ coronarium et $H$ capitatum.

Taxon $\begin{gathered}\text { Population } \\ \text { d'origine Désignation Phénotype }\end{gathered}$

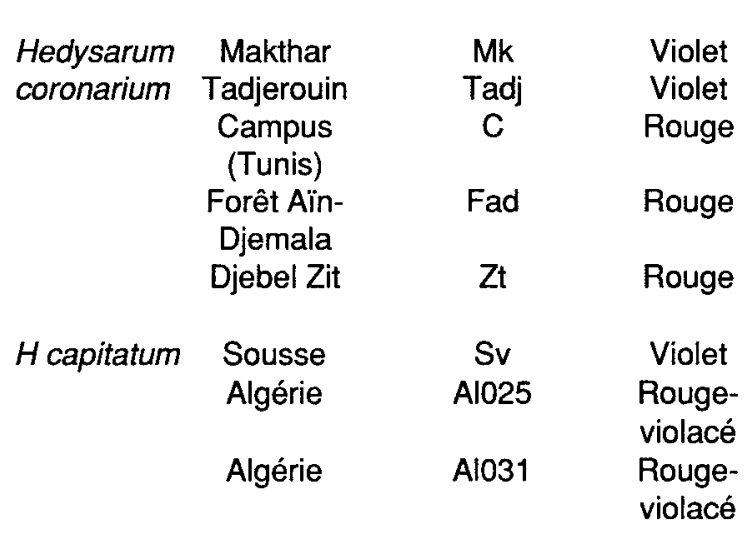


Pour connaitre la constitution pigmentaire des fleurs des différentes lignées utilisées dans cette analyse, nous nous sommes bornés à la détermination des anthocyanidines et des flavonols-aglycones. Pour cela, nous avons appliqué la technique de chromatographie bidimensionnelle sur couche mince de cellulose (Merck), déjà décrite par Nyborn (1964) et légèrement modifiée par Farcy et Cornu (1979).

Les solvants utilisés sont pour la première dimension : acide formique / $\mathrm{HCl}$ conc / eau (40/1/15), en volume; pour la deuxième dimension : alcool amylique / acide acétique / eau (2/1/1) en volume.

Cette technique, nous a permis d'une part, de séparer les composants des mélanges pigmentaires les plus complexes, et d'autre part, d'apprécier, par notation visuelle des plaques, les quantités relatives des différents pigments présents dans un même génotype. Cette appréciation a été confirmée, pour certaines lignées, par une détermination semi-quantitative des pigments floraux, réalisée dans le laboratoire du Professeur Cornu (INRA, Dijon), à l'aide d'un densitomètre (Vitatron).

\section{RÉSULTATS}

\section{Caractérisation biochimique des lignées parentales chez $\mathrm{H}$ coronarium}

L'application de la technique de chromatographie en deux dimensions nous a permis de définir la composition en flavonols et en anthocyanidines des deux phénotypes de base : fleurs violettes et fleurs rouges, rencontrés chez cette espèce (tableau II). Cinq pigments anthocyaniques ont été mis en évidence : la delphinidine, la pétunidine, la cyanidine, la malvidine et la paeonidine (fig 1). Trois types de flavonols ont été repérés : le kaempférol, la quercétine et la myricétine. Toutefois, la teneur des extraits en quercétine est nettement plus élevée que leur teneur en kaemptérol; la myricétine étant à l'état de traces. La différence phénotypique observée est due à une différence quantitative en anthocyanidines:

Les plantes à fleurs rouges se caractérisent par l'abondance de la paeonidine, la présence, en quantités plus faibles, de la cyanidine et de la malvidine, et, à l'état de traces, de la delphinidine et de la pétunidine.

Les plantes à fleurs violettes se caractérisent par la prédominance de la malvidine, à des concentrations nettement plus faibles, de la paeonidine et de la pétunidine, et, à l'état de traces, de la delphinidine et de la cyanidine.
Tableau II. Composition des fleurs en flavonols et en anthocyanidines chez $H$ coronarium et $H$ capitatum. $\mathrm{km}$ : kaempférol, Qu : quercétine, My : myricétine, Dp : delphinidine, $\mathrm{Pt}$ : pétunidine, $\mathrm{Mv}:$ malvidine, Cy : cyanidine, Pn : paeonidine. Le chiffre donné correspond à une appréciation visuelle quantitative de la tache obtenue (intensité et surface); la quantité la plus faible étant $t r$ (traces) et la plus forte 5 .

\section{Lignée Phénotype Flavonols Anthocyanidines $\mathrm{Km} Q u$ My Dp Pt Mv Cy Pn Tri-OH Di-OH}

$\begin{array}{llllllllll}\begin{array}{l}\text { H coronarium } \\ \text { Mk }\end{array} & \text { Violet } & 1 & 4 & \operatorname{tr} & \operatorname{tr} & 1 & 5 & \operatorname{tr} & 1 \\ \text { Tadj } & \text { Violet } & 1 & 3 & \operatorname{tr} & \operatorname{tr} & 1 & 4 & \operatorname{tr} & 1 \\ \mathrm{C} & \text { Rouge } & 1 & 3 & \operatorname{tr} & \operatorname{tr} & \operatorname{tr} & 1 & 1 & 5 \\ \text { Fad } & \text { Rouge } & 1 & 3 & \operatorname{tr} & \operatorname{tr} & \operatorname{tr} & 1 & 2 & 5 \\ \mathrm{Zt} & \text { Rouge } & 1 & 3 & \operatorname{tr} & \operatorname{tr} & \operatorname{tr} & 1 & 2 & 5\end{array}$

$H$ capitatum

$\begin{array}{llllllllll}\text { Sv } & \text { Violet } & 1 & 4 & \operatorname{tr} & \operatorname{tr} & 1 & 5 & \operatorname{tr} & 1 \\ \text { Al0258 } & \begin{array}{l}\text { Rouge- } \\ \text { violacé }\end{array} & 1 & 3 & \operatorname{tr} & 0,5 & 1 & 4 & 0,5 & 3,5 \\ \text { Al031 } & \begin{array}{l}\text { Rouge- } \\ \text { violacé }\end{array} & 1 & 3 & \operatorname{tr} & 0,5 & 1 & 4 & 0,5 & 3,5\end{array}$

II existe donc, chez $H$ coronarium, un certain équilibre entre les pigments non substitués en $5^{\prime}$ (paeonidine, cyanidine) et les pigments substitués en 5' (malvidine, pétunidine); la delphinidine étant toujours à l'état de traces. L'obtention du phénotype rouge serait le résultat du déplacement de cet équilibre vers le groupe d'anthocyanidines non substituées en $5^{\prime}$; alors que le phénotype violet serait la conséquence du déplacement de cet équilibre vers le groupe d'anthocyanidines substituées en 5 '.

\section{Caractérisation biochimique des lignées parentales chez $\mathrm{H}$ capitatum}

Trois lignées ont été analysées (tableau II): la lignée Sv, à fleurs violettes; les lignées Al025 et Al031, à fleurs rouge-violacées.

On constate que, pour les trois lignées utilisées, la classe des flavonols est représentée, comme c'est le cas pour $H$ coronarium, par le kaempférol (quantité faible), la quercétine (abondante) et la myricétine (à l'état de traces).

La composition des pétales en flavonols est donc la même quel que soit le phénotype. La différence entre phénotypes est plutôt due à une 
différence de composition quantitative en anthocyanidines :

- le phénotype violet se caractérise surtout par l'abondance de la malvidine; alors que les autres anthocyanidines, plus particulièrement la paeonidine et la cyanidine, sont nettement minoritaires;

- le phénotype rouge-violet correspond à une synthèse en quantités importantes et sensiblement égales de deux pigments anthocyaniques: la malvidine et la paeonidine. Les trois anthocyanidines identifiées à la delphinidine, à la pétunidine et à la cyanidine, sont là aussi minoritaires.

L'investigation des pigments floraux nous a ainsi permis de définir chimiquement les trois phénotypes de base, rencontrés chez les deux taxons : $H$ coronarium et $H$ capitatum. Tout en présentant la même composition qualitative en anthocyanidines, ces trois phénotypes se distinguent entre eux par la nature du (ou des) pigment(s) prépondérant(s). Le phénotype rouge se caractérise par l'abondance de la paeonidine, le rouge-violet par l'abondance simultanée de la paeonidine et de la malvidine, enfin le violet par la présence de la maldivine en quantité notable.

La distinction entre ces trois formes phénotypiques, notamment entre le violet et le rougeviolacé, a été confirmée par une analyse semiquantitative densitométrique des différents pigments présents (tableau III).

Si l'on tient compte de la structure moléculaire des différentes anthocyanidines mises en évidence chez Hedysarum (fig 1), on constate que la différence structurale majeure entre la malvidine et la paeonidine réside essentiellement dans la présence ou l'absence d'un groupement méthoxyle en $5^{\prime}$ au niveau du noyau benzénique latéral $\mathrm{B}$.

Or, il est actuellement admis que la substitution en $5^{\prime}$ des flavonoïdes est progressive (Braun, 1976; Kho, 1978; Jonsson et al, 1983;

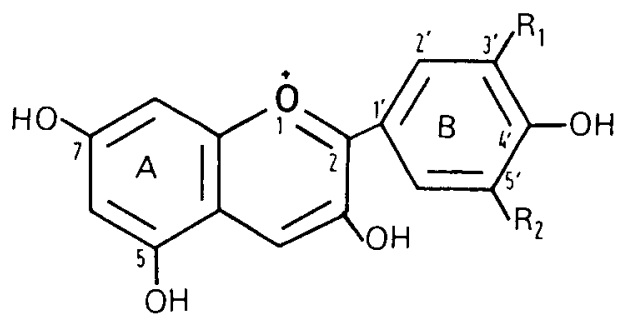

Fig 1. Anthocyanidines de $H$ coronarium ef $H$ capitatum. Cyanidine $\left(R_{1}=O H, R_{2}=H\right)$; Delphinidine $\left(R_{1}=R_{2}=O H\right)$; Paeonidine $\left(R_{1}=\mathrm{OCH}_{3} R_{2}=H\right)$; Pétunidine $\left(R_{1}=O C H 3\right.$, $\left.\mathrm{R}_{2}=\mathrm{OH}\right)$; Malvidine $\left(\mathrm{R}_{1}=\mathrm{R}_{2}=\mathrm{OCH}_{3}\right)$.

Cornu et Maizonnier, 1984) : ''hydroxylation en 5' intervient au niveau des dihydroflavonols, alors que les méthylations se produisent en fin de biosynthèse des molécules anthocyaniques.

Par conséquent, l'accumulation de pigments méthoxylés (paeonidine et malvidine) et la présence de la pétunidine en quantités faibles, aussi bien dans le phénotype violet que dans les phénotypes rouge ou rouge-violet, indiquent que les gènes contrôlant la méthylation fonctionnent efficacement dans les lignées parentales utilisées dans cette étude.

Ainsi, la diversité de la coloration florale observée chez Hedysarum est liée à une expression différentielle de gènes d'hydroxylation en 5 '. Le blocage de cette étape entraîne forcément celui de la méthylation en $5^{\prime}$ des molécules anthocyaniques. Si l'hydroxylation en 5' est bloquée, la dihydroquercétine (= dihydroflavonol dihydroxylé) se convertit en cyanidine, puis, par méthylation en 3 ', en paeonidine. Si l'hydroxylation en $5^{\prime}$ se réalise, la dihydroquercétine donne la dihydromyricétine (Kho, 1978). Ce flavonoïde trihydroxyle se convertit tout d'abord en delphinidine, puis, par méthylation en 3 ', en pétunidine. Par méthylation en 5 , cette anthocyanidine se transforme en malvidine.

Tableau III. Détermination semi-quantitative des anthocyanidines dans quelques lignées d'Hedysarum.

\begin{tabular}{|c|c|c|c|c|c|c|c|}
\hline \multirow{2}{*}{ Taxon } & \multirow{2}{*}{ Lignée } & \multirow{2}{*}{ Phénotype } & \multicolumn{5}{|c|}{ Anthocyanidines en \% } \\
\hline & & & $D p$ & $P t$ & $M v$ & $C y$ & $P n$ \\
\hline $\begin{array}{l}H \text { coronarium } \\
H \text { capitatum } \\
H \text { capitatum }\end{array}$ & $\begin{array}{l}\text { Mk } \\
\text { Al025 } \\
\text { Al031 }\end{array}$ & $\begin{array}{l}\text { Violet } \\
\text { Rouge-violet } \\
\text { Rouge-violet }\end{array}$ & $\begin{array}{l}1,95 \\
6,20 \\
5,42\end{array}$ & $\begin{array}{l}12,53 \\
17,00 \\
15,86\end{array}$ & $\begin{array}{l}69,65 \\
39,20 \\
38,11\end{array}$ & $\begin{array}{r}3,13 \\
8,50 \\
12,16\end{array}$ & $\begin{array}{l}12,74 \\
29,10 \\
28,44\end{array}$ \\
\hline
\end{tabular}




\section{Résultats des croisements rouge-violet $x$ violet chez $\mathrm{H}$ capitatum}

Nous avons réalisé deux types de croisements Al031 $\times$ Sv et Al025 x Sv. Les hybrides F1 issus de ces croisements sont homogènes et de phénotype violet; le pigment floral majeur étant la malvidine (tableau IV). Le caractère violet domine donc le caractère rouge-violet et l'abondance de la malvidine est due à un ou plusieurs gènes dominants.

En $F_{2}$, issue de l'autofécondation des individus $F_{1}$, les deux seuls phénotypes parentaux rougeviolet et violet sont observés. Les proportions relatives à chacune des deux formes phénotypiques sont assez semblables d'un type de croisement à l'autre.

Nous avons vérifié que, dans les différentes $F_{2}$ obtenues, les plantes de phénotype violet accumulent la malvidine, et celles de phénotype rouge-violet la malvidine et la paeonidine à la fois (tableau IV). Ceci prouve l'existence d'une corrélation très étroite entre la nature du pigment anthocyanique majeur et la coloration florale observée.

\section{Résultats des croisements rouge $x$ violet chez $\mathrm{H}$ coronarium}

Quatre types de croisements rouge $x$ violet ont été effectués (tableau $V$ ) dont trois ont servi également pour l'analyse biochimique des pigments floraux majeurs. Les résultats obtenus montrent que :

Tableau IV. Résultats des croisements violet $\mathrm{x}$ rougeviolet chez $\mathrm{H}$ capitatum.

Les résultats entre parenthèses indiquent le nombre de plantes dont la composition en anthocyanidines a été déterminée.

\begin{tabular}{|c|c|c|c|c|}
\hline Croisement & $\begin{array}{l}\text { Phénotype } \\
\text { en } F_{1}\end{array}$ & $\begin{array}{l}\text { notype } \\
F_{1} \\
\text { Pigment } \\
\text { majeur }\end{array}$ & \multicolumn{2}{|c|}{$\begin{array}{c}\text { Ségrégation } \\
\text { phénotypique en } F_{2}\end{array}$} \\
\hline $\mathrm{Al} 031 \times \mathrm{Sv}$ & Violet & Mv & $69(27)$ & $24(5)$ \\
\hline $\mathrm{Al} 025 \times \mathrm{Sv}$ & Violet & Mv & $113(41)$ & $26(10)$ \\
\hline
\end{tabular}

\footnotetext{
1 malvidine (Mv) accumulée; ${ }^{2}$ malvidine et paeonidine accumulées
}

- la $F_{1}$ est homogène et de phénotype violet caractérisé par l'accumulation de la malvidine; la synthèse de cette anthocyanidine est donc contrôlée par un ou plusieurs gènes dominants;

- la $F_{2}$ comporte trois phénotypes distinguables sans aucune ambiguité : rouge, violet, et rougeviolet, respectivement caractérisés par l'accumulation de la paeonidine, de la malvidine et des deux pigments à la fois.

Parallèlement à ces expériences d'hybridation, des croisements entre lignées de même phénotype ont été réalisés. Nous avons constaté que les croisements rouge $x$ rouge ou violet $x$ violet donnent lieu, en $F_{1}$ et en $F_{2}$, à des plantes à fleurs rouges ou violettes, respectivement.

\section{DISCUSSION}

Les principales caractéristiques des lignées utilisées dans cette analyse peuvent se résumer en deux alternatives : fleurs violettes / fleurs rougeviolacées, chez $H$ capitatum; fleurs violettes / fleurs rouges, chez $H$ coronarium.

L'observation des hybrides issus de croisements entre des lignées d'une même espèce montre que, dans chacune de ces alternatives, le caractère violet se transmettait comme un caractère dominant. Les nombreuses autofécondations des invidus $F_{1}$ qui ont été effectuées nous permettront de préciser le déterminisme génétique des caractères fleurs violettes - fleurs rouge-violacées chez $H$ capitatum et fleurs violettes - fleurs rouges chez $H$ coronarium.

Tableau V. Résultats des croisements rouge $x$ violet chez $H$ coronarium.

Les chiffres entre parenthèses indiquent le nombre de plantes dont la composition en anthocyanidines a été déterminée.

\begin{tabular}{|c|c|c|c|c|}
\hline \multirow[t]{2}{*}{ Croisement } & \multirow{2}{*}{$\begin{array}{c}\text { Phénotype } \\
\text { en } F_{1}\end{array}$} & \multicolumn{3}{|c|}{ Ségrégation } \\
\hline & & Violet $^{1}$ & $\begin{array}{l}\text { Rouge } \\
\text { violacée }\end{array}$ & Rouge 3 \\
\hline Fad $\times$ Mk & Violet1 & $196(58)$ & $48(9)$ & $24(8)$ \\
\hline $\mathrm{Mk} \times \mathrm{Zt}$ & Violet1 & 116 & 29 & 14 \\
\hline $\mathrm{C} \times \mathrm{Mk}$ & Violet1 & $168(73)$ & $34(13)$ & $13(6)$ \\
\hline Tadj $\times C$ & Violet1 & $173(67)$ & $47(21)$ & $18(7)$ \\
\hline
\end{tabular}

1 malvidine majeure; ${ }^{2}$ malvidine et paeonidine majeures; 3 paeonidine majeure 


\section{Hérédité de la coloration florale chez H capitatum}

Chacune des $F_{2}$, issues des croisements rougeviolet $x$ violet effectués chez ce taxon, montre une ségrégation phénotypique qui ne s'écarte pas significativement de la disjonction 3/1 (tableau VI). Ceci suggère que la souche à fleurs violettes diffère de chacune des souches à fleurs rouge-violacées par un seul couple d'allèles. L'allèle sauvage, dominant, doit entraîner une synthèse accrue de la malvidine seulement et détermine alors le phénotype violet. Par contre, l'allèle mutant, récessif, correspondrait à un équilibre malvidine paeonidine, déterminant ainsi le phénotype récessif rouge-violet.

Étant donné que la $F_{1}$ issue du croisement $\mathrm{Al}$ $025 \times$ Al031, montrait le phénotype rouge-violet (Baatout, communication personnelle), les mutations récessives impliquées seraient allèles.

En désignant par $V / v$, le couple d'allèles contrôlant l'hydroxylation en 5 ' des anthocyanidines chez $H$ capitatum, il devient clair que :

- le phénotype violet est dû à la présence du gène $V$, responsable d'une hydroxylation quasitotale en $5^{\prime}$ de la dihydroquercétine qui se convertit alors en dihydromyricétine, d'où l'accumulation de la malvidine;

- le phénotype rouge-violacée est dû à la présence, à l'état homozygote, du gène $v$ qui déterminerait une conversion partielle de la dihydroquercétine en dihydromyricétine. Ceci entraîne l'abondance simultanée de la paeonidine et de la malvidine.

\section{Hérédité de la coloration florale chez $\mathrm{H}$ coronarium}

L'observation des hybrides $F_{1}$ issus des croisements rouge $x$ violet (tableau $V$ ) montre que le phénotype violet, accumulant la malvidine, est déterminé par un ou plusieurs gènes dominants. Par ailleurs, l'absence de complémentation entre les lignées à fleurs rouges montre qu'elles sont homozygotes récessives pour les mêmes gènes.

En plus des phénotypes parentaux (rouge et violet), les différentes $F_{2}$, issues des croisements rouge $\mathrm{x}$ violet réalisés chez $H$ coronarium, montrent le phénotype rouge-violacé. Ceci nous amène à envisager l'intervention de deux couples d'allèles que nous pouvons désigner par $V / v$ et $R / r$. Dans ce cas, et étant donné que chacune de ces $F_{2}$ ne comprend que trois phénotypes seulement (tableau $\mathrm{VI}$ ), les gènes $V$ et $R$ doivent présenter une interaction de nature épistatique. II s'agit d'un cas d'épistasie dominante. En effet, les nombres observés dans ces $F_{2}$ peuvent être ajustés à la ségrégation théorique du type 12/3/1 (tableau VI).

En appelant $V$, le gène épistatique dominant et $R$, l'autre gène, les formules géniques seraient alors : $(V-R$ ou $V-r r)$ déterminant le phénotype violet; ( $(v R-)$ déterminant le phénotype rouge-violacé; ( $\mathrm{v} v \mathrm{rr}$ ) déterminant le phénotype rouge.

Étant donné que le croisement violet $\mathrm{x}$ violet fournit une $F_{2}$ sans ségrégation, les lignées à fleurs violettes doivent être homozygotes (VV).

Comme la $F_{2}$ issue des croisements rouge $x$ violet renferme des individus à fleurs rouge-

Tableau VI. Résultats des différents croisements et leur ajustement aux ségrégations théoriques. $V$ : violet, $R V$ : rouge-violet, $R=$ rouge.

\begin{tabular}{|c|c|c|c|c|c|c|c|c|c|}
\hline \multirow{2}{*}{ Croisement } & \multicolumn{3}{|c|}{ Ségrégation en $F_{2}$} & \multirow{2}{*}{$\begin{array}{l}\text { Rapport } \\
\text { théorique }\end{array}$} & \multicolumn{5}{|c|}{ Probabilité du } \\
\hline & $v$ & $R V$ & $R$ & & & & $\chi^{2}$ & & \\
\hline \multicolumn{10}{|l|}{$H$ capitatum } \\
\hline $\mathrm{Al} 025 \times \mathrm{Sv}$ & 113 & 26 & - & $3 / 1$ & 0,05 & $<$ & $\mathbf{P}$ & $<$ & 0,10 \\
\hline $\mathrm{Al} 031 \times \mathrm{Sv}$ & 69 & 24 & - & $3 / 1$ & 0,75 & $<$ & $\mathbf{P}$ & $<$ & 0,90 \\
\hline \multicolumn{10}{|l|}{$H$ coronarium } \\
\hline $\mathrm{C} \times \mathrm{Mk}$ & 168 & 34 & 13 & $12 / 3 / 1$ & 0,50 & $<$ & $\mathbf{P}$ & $<$ & 0,75 \\
\hline$M k \times Z t$ & 116 & 29 & 14 & $12 / 3 / 1$ & 0,25 & $<$ & $\mathbf{P}$ & $<$ & 0,50 \\
\hline Tadj x C & 173 & 47 & 18 & $12 / 3 / 1$ & 0,50 & $<$ & $P$ & $<$ & 0,75 \\
\hline Fad $\times$ Mk & 196 & 48 & 24 & $12 / 3 / 1$ & 0,10 & $<$ & $P$ & $<$ & 0,25 \\
\hline
\end{tabular}


violacées comportant, par conséquent, l'allèle $R$ qui ne peut provenir que des lignées à fleurs violettes, nous pouvons admettre les génotypes parentaux suivants : ( $\mathrm{V} \mathrm{rr}$ ) : génotype des lignées parentales à fleurs rouges; (VV RR) : génotype des lignées parentales à fleurs violettes.

Remarquons que l'hypothèse faisant intervenir deux gènes $V$ et $R$ dans le contrôle de l'hydroxylation en $5^{\prime}$ des anthocyanes chez $H$ coronarium, peut également être formulée pour expliquer les résultats observés chez $H$ capitatum. Dans ces conditions, on doit admettre les génotypes suivants : (VV RR) pour la souche Sv, à fleurs violettes; ( $v V R R$ ) pour les souches à fleurs rougeviolacées, Al025 et Al031.

\section{CONCLUSION}

L'étude de l'hérédité des anthocyanes florales chez $H$ coronarium et $H$ capitatum nous a permis de montrer que les couleurs rouge, rougeviolacée et violette des fleurs dépendent essentiellement des concentrations relatives des deux pigments : la paeonidine et la malvidine. D'autre part, cette analyse a prouvé l'intervention de deux gènes $V-v$ et $R-r$ dans le contrôle génétique de la pigmentation florale chez ces deux taxons. En combinant les résultats génétiques et biochimiques obtenus, nous pouvons faire correspondre à chaque lignée son génotype, sa composition en anthocyanidines et enfin sa coloration florale (tableau VII).

Le gène $V$ a une action sur la pigmentation des pétales en gouvernant la synthèse d'une

Tableau VII. Génotype, composition en anthocyanidines et phénotype des lignées utilisées dans l'étude de l'hérédité de la coloration florale chez $\mathrm{H}$ coronarium et $H$ capitatum. Dp : delphinidine, $\mathrm{Pt}$ : pétunidine, $\mathrm{Mv}$ : malvidine, $\mathrm{Cy}$ : cyanidine, Pn : paeonidine.

\begin{tabular}{|c|c|c|c|}
\hline & & $\begin{array}{l}\text { Composition en } \\
\text { anthocyanidines }\end{array}$ & $\begin{array}{l}\text { Coloration } \\
\text { florale }\end{array}$ \\
\hline & & $D p$ Pt $M v \quad C y$ & $P n$ \\
\hline
\end{tabular}

\begin{tabular}{|c|c|c|c|c|c|c|c|}
\hline$H$ coronarium & & & & & & & \\
\hline C Fad Zt & wrr & $\operatorname{tr}$ & $\operatorname{tr}$ & 1 & 1,5 & 5 & rouge \\
\hline H capitatum & & & & & & & \\
\hline Sv & VVRR & $\operatorname{tr}$ & 1 & 5 & $\operatorname{tr}$ & 1 & violet \\
\hline Al025, Al031 & vvRR & 0,5 & 1 & 4 & 0,5 & 3,5 & $\begin{array}{l}\text { rouge- } \\
\text { violacé }\end{array}$ \\
\hline
\end{tabular}

grande quantité de malvidine. Ainsi, nous pouvons penser que ce gène, en contrôlant l'hydroxylation en $5^{\prime}$ d'un flavonoïde précurseur, de la classe des dihydroflavonols (Harrison et Stickland, 1978; Kho, 1978), détermine la synthèse d'une anthocyanidine intermédiaire (= la pétunidine) qui se transforme en malvidine par méthylation en $5^{\prime}$. Étant donné que la pétunidine est invariablement détectée en faibles quantités en présence ou en absence de $V$, nous pouvons admettre que la formation de la malvidine est le résultat de l'activité d'un ou de plusieurs gènes supplémentaires favorisant la méthylation en $5^{\prime}$ de la pétunidine.

Quant au gène $R$, responsable du phénotype rouge-violet, il ne s'exprime qu'en l'absence du gène $V$, en l'occurrence dans les plantes de génotype vv (tableau VII). Le gène $R$ est donc hypostatique par rapport à $V$; son action se traduit, dans les plantes de génotype ( $v v$ ), par une synthèse assez limitée de malvidine. On aboutit alors dans ce phénotype rouge-violet à un équilibre entre les productions de paeonidine (non hydroxylée en $5^{\prime}$ ) et de malvidine (hydroxylée puis méthylée en $5^{\prime}$ ). La fonction du gène $R$ est donc une fonction hydroxylante (en position $5^{\prime}$ ) comme c'est le cas du gène $V$, mais moins importante. En présence de $V$, son activité n'est pratiquement pas décelable.

Ce gène $V$, par sa fonction hydroxylante en position $5^{\prime}$, nous rappelle des situations analogues décrites chez plusieurs espèces, notamment les gènes $R$ et $V$ respectivement de Salvia horminum (Cornu et Paynot, 1969) et Salvia splendens (Hendrychova-Tomkova, 1964), le gène Hf1 et Petunia hybrida (Meynet et al, 1971; Wiering et al, 1979; Cornu et Maizonnier, 1984) et le gène $L$ de Campanula medium (Braun, 1976). Par sa fonction, le gène $R$, identifié chez les taxons $H$ coronarium et $H$ capitatum, rapelle également le gène Hf2 de Petunia hybrida (Meynet et al, 1971; Wiering et al, 1979).

Bien que la fonction principale des gènes $V$ et $R$ soit une fonction d'hydroxylation en $5^{\prime}$, les anthocyanidines majeures mises en évidence dans les lignées à fleurs colorées utilisées dans cette étude sont la paeonidine, méthylée en 3 ', et la malvidine, diméthylée en $3^{\prime}$ et $5^{\prime}$. Ceci nous amène à admettre que les gènes contrôlant la méthylation, aussi bien en position 3' qu'en position $5^{\prime}$, soient dominants. Le contrôle génétique de la méthylation des anthocyanes mérite donc d'être analysé chez Hedysarum, en se basant notamment sur des mutants de gènes de méthylation. II est à signaler que, chez $H$ coronarium, 
des mutants à fleurs roses, accumulant la cyanidine, ont été repérés dans la population Fad (à fleurs rouges). En disposant actuellement de graines de ces mutants, il est possible d'étudier, entre autres, le contrôle génétique de la méthylation en 3 ' des anthocyanes chez cette espèce.

\section{REMERCIEMENTS}

A Cornu (INRA, Dijon) et M Marrakechi (Faculté des Sciences de Tunis) sont vivement remerciés pour leur fructueuse collaboration.

\section{RÉFÉRENCES}

Baatout H, Marrakechi M, Mathieu C, Vedel F (1985) Variation of plastid and mitochondrial DNAs in the genus Hedysarum. Theor Appl Genet 70, 577-584

Ballatore GP (1972) La produzione foraggera negli ambianti semiaridi, con particolar referimento alla Sicilia. Inf Agraria 27, 7453-7464

Braun A (1976) L'hérédité des anthocyanes florales. Ann Biol 15, 293-328

Cenni B, Jannella GG, Colombani N (1968) Chemical composition, digestibility and nutritive value of Sulla (Hedysarum coronarium L) hay produced in Volterra district. Ann Fac Med Vet Univ Pisa 20, 155-168

Chriki A, Harborne JB (1983) Anthocyanins of Hedysarum coronarium $\mathrm{L}$ and their contribution to flower colour variation. Phytochemistry (Oxf) 22, 23222323

Chriki A, Combes D, Marrakechi M (1982) Hérédité et analyse chromatographique de la pigmentation des fleurs chez l'espèce Hedysarum coronarium L. Agronomie 2, 915-922

Chriki A, Combes D, Marrakechi M (1984) Étude de la compétition pollinique chez le Sulla (Hedysarum coronarium L). Agronomie 4, 155-159

Chriki A, Combes D, Marrakechi M (1986) Contrôle génétique de la conversion des dihydroflavonols en anthocyanes chez l'Hedysarum coronarium Desf. CR Acad Sci 16, 585-588

Combes D, Espagnac H, Figier J (1975) Étude de populations naturelles d'Hedysarum coronarium $L$ du Nord de la Tunisie. Bull Soc Hist Nat Afr Nord 66, 107-122

Cornu A, Maizonnier D (1984) The genetics of Petunia. Plant Breed Rev 1, 11-58

Cornu A, Paynot M (1969) Hérédité et analyse chromatographique de la pigmentation anthocyanique des fleurs et bractées de Salvia horminum L. Ann Amélior Plant 19, 5-13

Farcy E, Cornu A (1979) Isolation and characterization of anthocyanin variants originating from the unstable system an2.1 in Petunia hybrida (Hort). Theor Appl Genet 55, 273-278

Figier J, Espagnac H, Combes D, Francillon G (1978) Mise en évidence de types morphologiques dans les populations naturelles de l'Hedysarum coronarium $L$ en Tunisie par analyse multivariable. $R e v$ Gén Bot 85, 21-62

Harborne JB (1973) Phytochemical methods. Chapman and Hall, London

Harrison BJ, Stickland RG (1978) Precursors and the genetic control of pigmentation. IV. Hydroxylation and methoxylation stages in anthocyanidin synthesis. Heredity 40, 127-132

Hendrychova-Tomkova J (1964) Genetic analysis of colour mutants in Salvia splendens. Preslia (Prague) $36,217-225$

Jonsson $L M V$, de Vlaming $P$, Wiering $H$, Aarsman MEG, Schram AW (1983) Genetic control of anthocyanin-o-methyltransferase activity in flowers of $\mathrm{Pe}$ tunia hybrida. Theor App/ Genet 66, 349-355

Kho KFF (1978) Conversion of hydroxylated and methylated dihydroflavonols into anthocyanins in a white flowering mutants of Petunia hybrida. Phytochemistry (Oxf) 17, 245-248

Meynet J, Cornu A, Paynot M (1971) Analyse du fonctionnement des gènes majeurs impliqués dans la pigmentation florale du Petunia. Ann Amélior Plant $21,103-116$

Monotti M (1975) Variabilita fenotipica tra ecotipi di Sulla (Hedysarum coronarium L). Genet Agrar 29, 163-178

Nybom N (1964) Thin layer chromatographic analysis of anthocyanidins. Physiol Plant 17, 154-164

Sarno R, Stringi L, Burgio G (1978) Relazioni intercorrenti tra alcuni caratteri biogranomici della Sulla (Hedysarum coronarium L). Quad Agron 9, 170-209

Trifi-Farah N, Chatti WS, Marrakechi M, Pernès $J$ (1989a) Déterminisme génétique de dix systèmes enzymatiques chez Hedysarum coronarium $\mathrm{L}$. Agronomie 9, 503-510

Trifi-Farah N, Chatti WS, Marrakechi M, Pernès J (1989b) Analyse de la variabilité morphologique et enzymatique des formes cultivées et spontanées d'Hedysarum coronarium L en Tunisie. Agronomie 9, 591-598

Wiering $\mathrm{H}$, de Vlaming $\mathrm{P}$, Cornu $\mathrm{A}$, Maizonnier $\mathrm{D}$ (1979) Petunia genetics. I. List of genes. Ann Amélior Plant 29, 611-622 